\title{
On The Energy Efficiency of Adaptive WBAN Systems for mHealth Services
}

\author{
M. Sudjai ${ }^{1, *}$, L.C. $\operatorname{Tran}^{2}$ and F. Safaei ${ }^{3}$ \\ ${ }^{1,2,3}$ School of Electrical, Computer, and Telecommunication Engineering (SECTE), University of Wollongong, NSW 2522,
}

Australia.

\section{Abstract}

We recently proposed an adaptive Wireless Body Area Network (WBAN) system for mHealth service, which shows better
bit error performance compared to the non-adaptive one. However, analysis and maximization of the energy efficiency as
one of the key design considerations of these networks remain an open problem. Therefore, this paper investigates the
energy efficiency of the proposed adaptive WBAN system by considering both the transmission power and the power
consumed in the circuitry which consists of rate-independent and rate-dependent components. Since the distance between
the hub and the wireless access point in WBAN is relatively short, beside the transmission power, the circuitry power also
plays an important role. The adaptive WBAN is evaluated in two different multiple input multiple output configurations. It
is found that the adaptive WBAN system substantially outperforms the non-adaptive one in terms of energy efficiency in
both $2 \mathrm{I} 1 \mathrm{O}$ and $2 \mathrm{I} 2 \mathrm{O}$ configurations. Furthermore, the $2 \mathrm{I} 2 \mathrm{O}$ adaptive WBAN system is 7.9 dB superior to the $2 \mathrm{I} 1 \mathrm{O}$ in terms
of energy efficiency. This is because the $2 \mathrm{I} 2 \mathrm{O}$ configuration has a higher diversity order and a better array gain compared
to the $2 \mathrm{I} 1 \mathrm{O}$ configuration, thus the former has better capability to mitigate fading and improve the link reliability. This
energy related performance coupled with the improved bit error rate performance suggests that the proposed adaptive
WBAN scheme is an attractive physical layer option of a WBAN system for mHealth services. Thus, this scheme could
provide not only better quality of services, but also energy saving to enhance the battery life of a WBAN system.

Keywords: Adaptive, Energy Efficiency, MIMO, WBAN.

Received on 28 July 2016, accepted on 22 September 2016, published on 21 March 2017

Copyright (C) 2017 M. Sudjai et al., licensed to EAI. This is an open access article distributed under the terms of the Creative Commons Attribution licence (http://creativecommons.org/licenses/by/3.0/), which permits unlimited use, distribution and reproduction in any medium so long as the original work is properly cited.

doi: 10.4108/eai.21-3-2017.152391

\section{Introduction}

Wireless Body Area Networks (WBAN) potentially reduce the healthcare costs by facilitating mobile health (mHealth) services [1]. WBAN allows connections of various bodily sensors to monitor and extract physiological data and relay the data wirelessly to a data or monitoring center, such as the doctor's terminal [1-2]. Due to the nature of body centric propagation and health issues such as the Specific Absorption Rate (SAR), the communication system and bodily sensors devices have to be small, lightweight, robust and energy-efficient [1-3]. Moreover, because future applications require better quality of service and data rates, the WBAN system should be capable to provide higher capacity [1], [4].
In order to support those WBAN features, the optimized adaptive Space-Time-Frequency Coded (STFC) Multi-Band Orthogonal Frequency Division Multiplexing Ultra-Wideband (MB-OFDM UWB) system as a WBAN physical layer was proposed in [5-7]. For simplicity, let us refer to this proposal as the optimized adaptive WBAN systems hereafter. It was shown in [6] and [7] that the optimized adaptive WBAN scheme provides a substantial performance improvement compared to a non-adaptive one. Readers may refer to [57] for more details about the adaptive WBAN systems.

Recent efforts to minimize energy consumptions, hence increasing energy efficiency, in a wireless communication system are numerous, including in wireless sensor networks [8-13] and in WBAN [14-21]. However, analysis and maximization of the energy efficiency, while 
maintaining a satisfactory level of performance, as one of the key design considerations of the proposed adaptive WBAN system have not been found in the literature.

Therefore, this paper investigates the energy efficiency of our previously proposed adaptive WBAN system (as described in [7]), in both $2 \mathrm{I} 1 \mathrm{O}$ (2 transmitter and 1 receiver antennas) and $2 \mathrm{I} 2 \mathrm{O}$ ( 2 transmitter and 2 receiver antennas) configurations. It is important to highlight the difference between this paper and the work in [7]. [7] described our proposed adaptive WBAN system, without considering its energy consumption. While, this paper focus on the energy efficiency investigation of such system. This paper considers not only the transmission power, but also the power consumed in the circuitry. The circuitry power consists of the rate-independent power consumptions, e.g. power consumed in the transceiver's components, such as mixer, filter, $\mathrm{ADC} / \mathrm{DAC}$, and the rate-dependent power consumption in encoders and decoders, including STFC encoders and decoders. The energy efficiency of the adaptive WBAN system is evaluated as a function of distance between a hub and a Wireless Access Point (WAP) in comparison with the non-adaptive one in the same environment. It is found that the optimized adaptive WBAN system substantially outperforms the non-adaptive one in terms of energy efficiency. Furthermore, the relation between energy efficiency and spectral efficiency of the adaptive WBAN system is also investigated. In this case, the $2 \mathrm{I} 2 \mathrm{O}$ configuration provides a superior performance in terms of energy efficiency, compared to the $2 \mathrm{I} 1 \mathrm{O}$ configuration, at any distance due to its higher diversity order and its array gain.

The major contribution of this paper is the analysis and maximization of energy efficiency of the proposed optimized adaptive WBAN system compared to the nonadaptive one. The proposed optimized adaptive WBAN system has been fully described in [7]. Due to the limited space, a short overview of this system will be mentioned in Section III of this paper. Readers may refer to [7] for more detail.

The paper is organized as follows. Section 2 describes related works during past years. Section 3 provides an overview of the proposed adaptive WBAN system mentioned in [5-7]. The analysis of the energy efficiency of this system is provided in Section 4. Section 5 describes the relation between energy efficiency and spectral efficiency of the adaptive WBAN system. Numerical evaluations are given in Section 6. Finally, Section 7 concludes the paper.

\section{Related Works}

Due to the nature of short range communication systems owing to limited power sources, energy efficiency plays a pivotal role in the networks design considerations [1, 8-21]. Numerous recent works focus on this issue, including in wireless sensor networks and WBAN. For instance, authors in [8] investigated the modulation strategy to minimize the total energy consumption required, given throughput and delay requirements as the constraints. In [9] a general model was proposed to optimize energy efficiency and spectral efficiency over a flat-fading channel. It considers all power consumed in transmission and circuitry including rate-dependent circuit power. However, it does not take into consideration a frequency selective fading. Amin, et.al. proposed a multi-objective optimization between energy efficiency and spectral efficiency of an adaptive power loading problem in a general OFDM system [10]. However, the performance resulted from this optimization was sensitive to channel estimator errors. Authors in [11] investigated the energy efficiency of a per-subcarrier antenna selection of MIMO OFDM systems with linear scaling. They utilized a data allocation strategy and a peak power reduction to optimize the trade-off between energy efficiency and capacity. An effort to take advantage of a rich diversity of multipath fading channel was investigated in [12]. It proposed an energy consumption model of MIMO and cooperative MIMO wireless sensor networks that takes into account power consumed in both transmission and circuitry. A cross layer adaptive modulation intended to reduce the transmission energy in wireless sensor networks was proposed in [13].

The energy efficiency issue in the WBAN communication system has been covered by many researchers such as in [14-21]. For instance, the energy efficiency and reliability of a wireless biomedical implant system were evaluated in [14] in order to introduce an augmentation protocol for the physical layer of the medical implant communication service standard for implant WBAN nodes. Authors in [15] investigated an energy-efficient cooperative relay selection scheme for ultra-wideband WBANs. However, the scheme was not suitable for all distances and relay positions. The problem of optimal power allocation with the constraint of a targeted outage probability in a cooperative WBAN was examined in [16]. This work also considers the impact of posture and movement of a human body on the performance. Authors in [17] proposed an energy efficient WBAN by selecting the appropriate sensors to activate based on some knowledge about the disorders to detect. Authors in [18] proposed an energy efficient MAC protocol designed by a flexible bandwidth allocation to improve a node's energy efficiency. However, it did not provide a detail energy analysis including energy consumed in transmission and circuitry. The energyefficient TDMA-based MAC protocol for WBAN considering a static or fixed topology of the network was investigated in [19]. Nonetheless, it did not consider the movement of WBAN nodes including the body directions toward external access points. Authors in [20] examined the energy efficiency and delay parameters as the performance metrics of dual physical layers WBAN and the design of MAC protocols to optimize data forwarding between those layers to the external networks. In [21] an 
energy-aware WBAN network design model was proposed by adopting a routing and relay positioning mechanism to achieve higher energy efficiency and lower installation cost.

All above approaches do not consider a very dispersive CM4 (Channel Model 4) WBAN channel with body movement consideration. Also the rich diversity of WBAN channels has not been utilized for energy consumption reduction through an adaptive MIMO WBAN approach. In addition, analysis and maximization of the energy efficiency of the proposed adaptive WBAN system, while maintaining a satisfactory level of performance, have not been found in the literature. Thus, this paper addresses these problems in order to demonstrate that the proposed adaptive WBAN scheme is not only capable of providing an improved bit error performance, but also able to generate better energy efficiency compared to the non-adaptive one. These features are vital for enhancing a WBAN network's lifetime while maintaining a good level of service quality.

\section{Adaptive WBAN Systems}

\subsection{Adaptive WBAN Scenario}

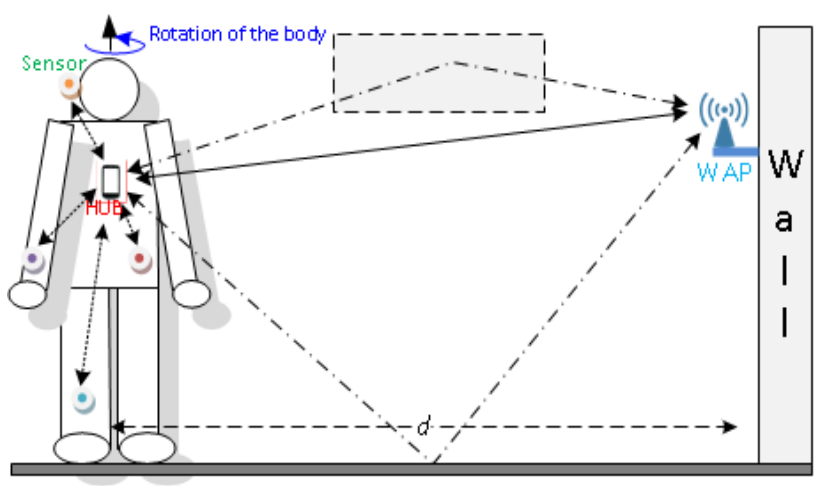

Figure 1. Adaptive WBAN Scenario [7]

Let us assume a hub is positioned in front of body (in torso). This hub is connected to various implantable and wearable body sensors via channel model 2 (CM2) and channel model 3 (CM3) channels. CM2 is a channel model characterizing an implant-to-body surface and implant-to-external links [22]. CM3 defines a body surface-to-body surface link for both line of sight (LOS) and non-line of sight (NLOS) links [22]. The hub is connected to an external fixed WAP placed on the wall through a CM4 channel at the distance $d$. CM4 is intended to stream a huge amount of data from various inand on-body sensors collected by the hub to an external processing centre such as a doctor's monitoring device through a WAP. The random movement of the body causes random body direction, in which the sensors and hub are placed, toward external WAP. This leads to the random channel behaviors. Thus, the CM4 channel characteristics will vary according to the body direction toward the access point [22]. To maintain a targeted performance, three adaptive schemes are applied to different channel fading conditions experienced by the rotating transceiver of the hub on the body [5-7].

\subsection{Review of the Proposed Adaptive WBAN System Model}

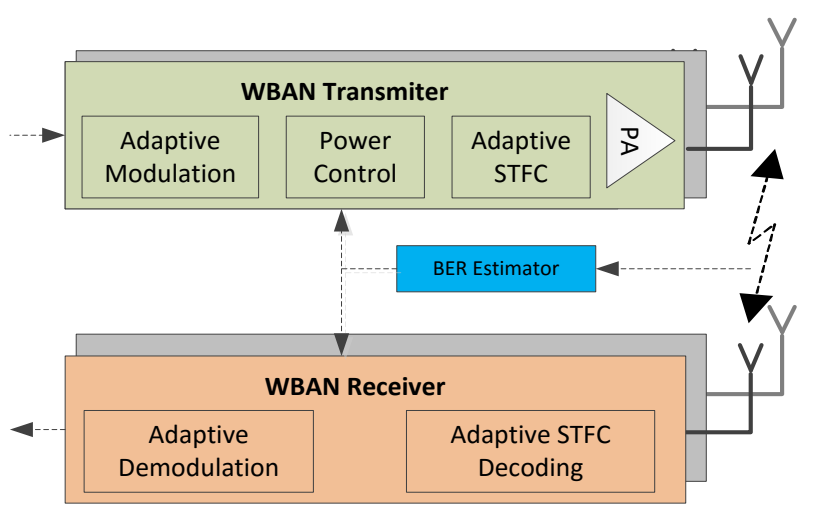

Figure 2. Adaptive WBAN Transceiver.

The proposed adaptive WBAN system model [5-7] employs a MIMO configuration with $M_{T}$ transmit antennas and $M_{R}$ receive antennas as shown in Figure 2. The transmitter consists of an adaptive modulation, power control, and an adaptive STFC block that controls three possible sets of modulation, signal power, and STFC coding, referred to as $\operatorname{Set}-i(i=1,2,3)$. The STFC utilizes either a full rate (rate 1) Alamouti code [23] or a high rate (rate 3/2) Sezginer-Sari code [24]. The benchmark for comparative performance assessment is the non-adaptive WBAN system employing QPSK modulation, STFC rate 1.0 , and normalized power 1.0 (thus providing a $2 \mathrm{bps} / \mathrm{Hz}$ spectral efficiency). Set- 1 is aimed for the best channel in order to maximize the throughput by using QPSK, STFC rate $3 / 2$ and power 1.5 , resulting in a $3 \mathrm{bps} / \mathrm{Hz}$ spectral efficiency. Set-2 is employed for the average quality channel, hence employing the same scheme as the nonadaptive system, i.e. using QPSK, STFC rate 1, and power 1. Set-3 is aimed to tackle the worst channel by employing BPSK modulation, STFC rate 1, and power 0.5 resulting in $1 \mathrm{bps} / \mathrm{Hz}$ spectral efficiency. The patient turns the body randomly over time, thus it is reasonable to assume that the body direction toward the access point is totally random, resulting in the equi-probability of the occurrence of Set-i. Therefore, by assuming that this condition is hold (i.e., the Set-i occurrence is equiprobable), thus this adaptive scheme arrangements maintain the average spectrum efficiency (i.e., $2 \mathrm{bps} / \mathrm{Hz}$ ) and the average normalized power (i.e., power 1.0) 
exactly the same as those in the non-adaptive system (2 $\mathrm{bps} / \mathrm{Hz}$ and power 1.0 , respectively).

Table 1. Parameters of the adaptive scheme

\begin{tabular}{ccccc}
\hline \hline Set-i & Modulation & $\begin{array}{c}\text { Norm. } \\
\text { Power }\end{array}$ & $\begin{array}{c}\text { STFC } \\
\text { coding rate }\end{array}$ & $\begin{array}{c}\text { Spectral } \\
\text { efficiency } \\
\text { (bps/Hz) }\end{array}$ \\
\hline Set-1 & QPSK & 1.5 & 1.5 & 3 \\
Set-2 & QPSK & 1.0 & 1 & 2 \\
Set-3 & BPSK & 0.5 & 1 & 1 \\
\hline
\end{tabular}

The frame structure comprises of two portions. At the first $f$ portion of the frame, $(0<f<1)$, Set- 2 is selected as the default scheme. The remaining portion of the frame employs adaptive scheme Set-i according to the adaptive algorithm. During each frame transmission, the receiver measures the signal quality at the receive antenna, i.e., BER as the metric for the adaptation, in a portion $f$ of the frame. This BER is not fed back to the transmitter, but is compared to the pre-set upper and lower thresholds according to the algorithm mentioned in the next subsection to decide one out of three possible sets of adaptive schemes to be employed in the remaining portion of the frame. Then, the receiver sends two bits to the transmitter via a feedback link to inform the transmitter about which set of adaptive schemes should be used in the transmission of the remaining portion of the frame. As aforementioned, the three sets are equi-probable, hence the average spectral density and average total transmitted power in the adaptive system are equal to those in a nonadaptive system, for a fair comparison. Since the first portion of the frame in both adaptive and non-adaptive system employ the same scheme (Set-2) [5], therefore for a comparison purpose, we only consider the adaptive scheme used in the remaining portion of the frame in this energy efficiency analysis.

Suppose $\overline{\mathrm{x}}=\left[x_{1}, x_{2}, \ldots, x_{N_{f f t}}\right]^{T}$ be an OFDM symbol vector, where $N_{f f t}$ is the FFT/IFFT size. The power control block adjusts the transmit power and the adaptive STFC block creates a space-time code either with a full rate or a 3/2-rate based on the feedback information from the receiver. The full rate code, i.e. the Alamouti code [23], converts two consecutive symbol vectors $\overline{\mathrm{x}}_{1}$ and $\overline{\mathrm{x}}_{2}$ into a STFC block

$$
\mathbf{X}=\left\{\overline{\mathrm{x}}_{t, m}\right\}_{T \times M}=\left[\begin{array}{cc}
\overline{\mathrm{x}}_{1} & \overline{\mathrm{x}}_{2} \\
-\overline{\mathrm{x}}_{2}^{*} & \overline{\mathrm{x}}_{1}^{*}
\end{array}\right]
$$

where $(\cdot)^{*}$ denotes complex conjugate, $t$ indicates time slot and $m$ indicates the $m^{\text {th }}$ transmit antenna. $\overline{\mathrm{x}}_{1}$ and $\overline{\mathrm{x}}_{2}$ are symbol vectors transmitted from the first and the second antenna at one time slot, respectively, then followed by symbol vectors $-\bar{x}_{2}^{*}$ and $\bar{x}_{1}^{*}$ at the next consecutive time slot. For a 3/2-rate STFC, the symbol vectors are encoded following the Sezginer-Sari code [24]
$\mathbf{X}=\left\{\overline{\mathrm{x}}_{t, m}\right\}_{T \times M}=\left[\begin{array}{cc}a \overline{\mathrm{x}}_{1}+\frac{b \overline{\mathrm{x}}_{3}}{\sqrt{2}} & -\left(c \overline{\mathrm{x}}_{2}^{*}+\frac{d \overline{\mathrm{x}}_{3}^{*}}{\sqrt{2}}\right) \\ a \overline{\mathrm{x}}_{2}+\frac{b \overline{\mathrm{x}}_{3}}{\sqrt{2}} & c \overline{\mathrm{x}}_{1}^{*}+\frac{d \overline{\mathrm{x}}_{3}^{*}}{\sqrt{2}}\end{array}\right]$

$\overline{\mathrm{x}}_{3}$ is the third symbol vector. The vectors $a \overline{\mathrm{x}}_{1}+\frac{b \overline{\mathrm{x}}_{3}}{\sqrt{2}}$ and $-\left(c \overline{\mathrm{x}}_{2}^{*}+\frac{d \overline{\mathrm{x}}_{3}^{*}}{\sqrt{2}}\right)$ are transmitted from the first and the second antenna at one time slot, respectively, then followed by the symbol vectors $a \overline{\mathrm{x}}_{2}+\frac{b \overline{\mathrm{x}}_{3}}{\sqrt{2}}$ and $c \overline{\mathrm{x}}_{1}^{*}+\frac{d \overline{\mathrm{x}}_{3}^{*}}{\sqrt{2}}$ at the next consecutive time slot. $a, b, c$, and $d$ are complex-valued design parameters. The optimal parameters $a=c=\sqrt{2}$, and $b=d=(1+j \sqrt{7}) / 4$ as determined in [24] are utilized. The received signals can be written in a matrix form $\mathcal{R}$ as

$$
\mathcal{R}=\mathbf{X} \circ \mathcal{H}+\mathcal{N}
$$

where $\mathcal{H}$ is the FFT transform of the channel matrix $\boldsymbol{h}$, and the operation (o) denotes the matrix multiplication similarly to the conventional matrix multiplication, except that each entry in $\mathcal{R}$ and $\mathcal{H}$ is not a single number, but a vector. The detected vectors are decided by the following Maximum Likelihood (ML) rule

$$
\left\{\tilde{\mathrm{x}}_{t, m}\right\}=\arg \min _{\left\{\overline{\mathrm{x}}_{t, m}\right\}}\|\mathcal{R}-\mathbf{X} \circ \mathcal{H}\|_{F}^{2}
$$

Readers interested in a full signal analysis of this adaptive WBAN system may refer to [5-7].

\subsection{Adaptive Algorithm}

As aforementioned, the adaptive scheme is controlled by the measured BER and the BER thresholds. Two BER thresholds, i.e. upper threshold $B E R_{U}$ and lower threshold $B E R_{L}$, are defined that determine the selection of one among three possible Set-i. The upper and lower thresholds are derived from the average non-adaptive BER performance as the benchmark. The thresholds are linearly defined to reflect a constant deviation in the whole range of SNR w.r.t. to the non-adaptive average BER performance as the reference point. The detail adaptive algorithm can be found in in [7].

\section{Energy Efficiency Analysis of Adaptive WBAN Systems}

The energy efficiency associated with the employed adaptive scheme Set-i, $i \in\{1,2,3\}$, is considered as the ratio between total power consumption and total data rate or traffic in that scheme [8]

$$
E_{i}=\frac{P_{T o t, i}}{R_{i}} \quad\left(\frac{\text { Joule }}{\text { bit }}\right)
$$


where $P_{T o t, i}$ and $R_{i}$ are the total power consumption and data rate (or traffic) in the scheme Set-i, respectively. Note that $R_{i}$ is equal to $B \Gamma_{i}$ where $\Gamma_{i}$ is the spectral efficiency (or normalized capacity) in bps/Hz of the scheme Set- $i$ and $B$ is the system bandwidth. The total power consumed during transmission of the data rate $R_{i}$ consists of the power dissipated in the circuitry $P_{C, i}$ and power consumed in the power amplifier (PA) $P_{P A, i}$. Let us assume that $P_{C}$ comprises a rate-independent power $P$, which is consumed in the parts of the transceiver, including adaptive modulation and power control, and a rate-dependent power $P\left(R_{i}\right)$ that is dissipated e.g. in the channel encoder/decoder and STFC encoder/decoder. $P\left(R_{i}\right)$ is assumed to increase linearly with $R_{i}$, i.e. $P\left(R_{i}\right) \sim \vartheta R_{i}$, where $\vartheta$ is a constant [9]. Then circuitry's power is

$$
P_{C, i}=P+\vartheta R_{i}
$$

The power dissipated in the amplifier in order to transmit the data rate $R_{i}$ is

$$
P_{P A, i}=(\xi / \eta) \times P_{T, i}
$$

where $\xi$ is the peak-to-average power ratio (PAPR), $\eta$ is the drain efficiency of the power amplifier, and $P_{T, i}$ is the average transmit power. Then the total power consumption is

$$
P_{T o t, i}=P_{C, i}+P_{P A, i}=P+\vartheta R_{i}+(\xi / \eta) \times P_{T, i}
$$

For simplicity, it is assumed that the path loss model between transmitter and receiver associated with the scheme Set-i $P L_{i}$ follows a log normal distribution [25]

$$
P L_{i}(d B)=P L_{o}+10 n_{i} \log _{10} \frac{d}{d_{o}}+S_{i}
$$

where $P L_{o}$ is the path loss at the reference point $d_{o}, n_{i}$ is the path loss exponent of the link, and $S_{i}$ is the shadowing component of the link which is a zero mean Gaussian random variable with a standard deviation $\sigma_{i}$. The instantaneous signal to noise ratio (SNR) at the receive antenna is $\beta_{i}=\frac{P_{R, i}}{N_{i}}$, where $P_{R, i}$ is the received signal power, and $N_{i}=N_{o} B N_{f}$ is the total noise power at the receiver in this link [25]. $N_{o}=174 \mathrm{dBm} / \mathrm{Hz}$ is the two sided noise spectral density [26], and $N_{f}$ is the noise Figure. If $\bar{\beta}_{i}$ is an average SNR at the receive antenna to achieve a targeted symbol error rate (SER) in the link- $i$, then the average transmit power $\bar{P}_{T, i}$ to satisfy this requirement is

$$
\bar{P}_{T, i}=\bar{P}_{R, i}\left(\frac{10^{\left(\frac{P L_{o}}{10}\right)}}{d_{o}^{n_{i}}}\right) d^{n_{i}} 10^{\left(\frac{S_{i}}{10}\right)}
$$

From (5), (8), and (10), the energy efficiency of the link- $i$ $E_{i}$ is as follow

$$
E_{i}=\frac{P+\vartheta R_{i}}{R_{i}}+(\xi / \eta) \frac{\bar{P}_{R, i}}{R_{i}}\left(\frac{10^{\left(\frac{P L_{o}}{10}\right)}}{d_{o}^{n_{i}}}\right) d^{n_{i}} 10^{\left(\frac{S_{i}}{10}\right)}
$$

The first part of $E_{i}$ is the circuitry energy consumption per bit, and the second part is the transmission energy per bit. With little manipulation, (11) can be rewritten as a function of $\bar{\beta}_{i}$

$$
E_{i}=\frac{P+\vartheta R_{i}}{R_{i}}+(\xi / \eta) \frac{\bar{\beta}_{i} N_{o} B N_{f}}{R_{i}}\left(\frac{10^{\left(\frac{P L_{o}}{10}\right)}}{d_{o}^{n_{i}}}\right) d^{n_{i}} 10^{\left(\frac{S_{i}}{10}\right)}
$$

As aforementioned, the adaptive WBAN system employs three sets of adaptive schemes, Set-i, $i \in\{1,2,3\}$. Those adaptive schemes will be assessed with $2 \mathrm{I} 1 \mathrm{O}$ and 2I2O MIMO configurations. Let us assume that perfect channel knowledge is known at the receiver, but unknown to the transmitter. A maximum likelihood detector is employed at the receiver. The SNR $\gamma_{i}$ at the input of detector is [27]

$$
\gamma_{i}=\frac{1}{M} \sum_{m=1}^{M}\left|h_{m}\right|^{2} \beta_{i}
$$

where $M$ is the maximum number of channels between the transmitter and the receiver, and $h_{m}$ is the channel gain. The symbol error probability $P_{e, i}$ at the detector is [26], [27]

$$
P_{e, i}=\bar{N}_{e, i} Q\left(\sqrt{\frac{\gamma_{i} d_{\min , i}^{2}}{2}}\right)
$$

where $\bar{N}_{e, i}$ is the number of neighborhoods in the modulation constellation, and $d_{m i n, i}$ is the minimum Euclidean distance of the signal constellation, respectively. By applying a Chernoff bound, the upper bound of (14) is obtained as follows [27]

$$
P_{e, i} \leq \bar{N}_{e, i} e^{-\left(\sum_{m=1}^{M}\left|h_{m}\right|^{2}\right) \beta_{i} \frac{d_{m i n, i}^{2}}{4 M}}
$$

Assuming $h_{m}$ is an independent zero-mean circulant symmetric complex Gaussian (ZMCSCG), then the average symbol error probability is $\bar{P}_{e, i}=\mathcal{E}\left\{P_{e, i}\right\}$ which is upper bounded by [27]

$$
\bar{P}_{e, i} \leq \bar{N}_{e, i} \prod_{i=1}^{M} \frac{1}{1+\frac{\beta_{i} d_{m i n}^{2} i}{4 M}}
$$




\subsection{I10 configuration}

In this configuration, the numbers of transmit antennas $M_{T}$ and receive antennas $M_{R}$ are equal to 2 and 1 , respectively. Thus, the maximum number of channels between transmitter and receiver is $M=M_{T}=2$. In a high SNR region $\left(\beta_{i} \gg 1\right)$, then

$$
1+\frac{\beta_{i} d_{\min , i}^{2}}{4 M} \approx \frac{\beta_{i} d_{\min , i}^{2}}{4 M}
$$

therefore the average symbol error probability of this MIMO configuration in (16) becomes

$$
\bar{P}_{e, i} \leq \bar{N}_{e, i}\left(\frac{\beta_{i} d_{\min , i}^{2}}{8}\right)^{-2}
$$

\section{Set-1 and Set-2}

Set-1 utilizes a QPSK modulation and a Sezginer-Sari code [24], and Set-2 employs a QPSK modulation and an Alamouti code [23]. In both sets, $\bar{N}_{e, i}=2$, and $d_{\text {min }, i}=$ $\sqrt{2}$. Therefore, from (17) the upper bound of symbol error probability is

$$
\bar{P}_{e, i} \leq 2\left(\frac{\left(\frac{\bar{P}_{R, i}}{R_{i}}\right)}{4 N_{o}}\right)^{-2}
$$

where $i \in\{1,2\}$. Therefore, the average power at the receive antenna required to achieve a target SER $\bar{P}_{e, i}$ is

$$
\bar{P}_{R, i}=\frac{4 R_{i} N_{o}}{\sqrt{0.5 \bar{P}_{e, i}}}
$$

By substituting (19) in (11), the energy efficiency for Set1 and Set-2 in this $2 \mathrm{I} 1 \mathrm{O}$ configuration for the target SER $\bar{P}_{e, i}$ is

$$
E_{i=1,2}=\frac{P+\vartheta R_{i}}{R_{i}}+(\xi / \eta) \frac{4 N_{o}}{\sqrt{0.5 \bar{P}_{e, i}}}\left(\frac{10^{\left(\frac{P L_{o}}{10}\right)}}{d_{o}^{n_{i}}}\right) d^{n_{i}} 10^{\left(\frac{S_{i}}{10}\right)}
$$

\section{Set-3}

Set-3 employs a BPSK modulation and an Alamouti code [23]. It is assumed that an antipodal signal is used for the BPSK modulation. This means $\bar{N}_{e, i}=1$ and $d_{\text {min }, i}=2$. In a high SNR region $\left(\beta_{i} \gg 1\right)$, the upper bound of the average probability of symbol errors is

$$
\bar{P}_{e, i} \leq\left(\frac{\left(\frac{\bar{P}_{R, i}}{R_{i}}\right)}{2 N_{o}}\right)^{-2}
$$

Here, $i \in\{3\}$. The average received power required for the target SER $\bar{P}_{e, i}$ is

$$
\bar{P}_{R, i}=\frac{2 R_{i} N_{o}}{\sqrt{\bar{P}_{e, i}}}
$$

Energy efficiency of Set-3 in this MIMO configuration can be found by applying (22) to (11)

$E_{i=3}=\frac{P+\vartheta R_{i}}{R_{i}}+(\xi / \eta) \frac{2 N_{o}}{\sqrt{\bar{P}_{e, i}}}\left(\frac{10^{\left(\frac{P L_{o}}{10}\right)}}{d_{o}^{n_{i}}}\right) d^{n_{i}} 10^{\left(\frac{S_{i}}{10}\right)}$

\section{2. $2 \mathrm{I} 2 \mathrm{O}$ configuration}

In this configuration, the maximum number of links between transmitter and receiver $M=M_{T}=M_{R}=2$. In high SNR region $\left(\beta_{i} \gg 1\right)$ the average symbol error probability becomes [27]

$\bar{P}_{e, i} \leq \bar{N}_{e, i}\left(\frac{\beta_{i} d_{\text {min }, i}^{2}}{4 M_{T}}\right)^{-M_{T} M_{R}} \leq \bar{N}_{e, i}\left(\frac{\beta_{i} d_{\min , i}^{2}}{8}\right)^{-4}$

\section{Set-1 and Set-2}

Using the same method mentioned in Section 4.1, the upper bound of symbol error rate is

$$
\bar{P}_{e, i} \leq 2\left(\frac{\left(\frac{\bar{P}_{R, i}}{R_{i}}\right)}{4 N_{o}}\right)^{-4}
$$

where $i \in\{1,2\}$. Thus, the average power at the receiver antenna required to achieve the target SER $\bar{P}_{e, i}$ in these scheme is

$$
\bar{P}_{R, i}=\frac{4 R_{i} N_{o}}{\sqrt[4]{0.5 \bar{P}_{e, i}}}
$$

By replacing (26) in (11), the energy efficiency for Set-1 and Set-2 in this $2 \mathrm{I} 2 \mathrm{O}$ configuration is

$$
E_{i=1,2}=\frac{P+\vartheta R_{i}}{R_{i}}+(\xi / \eta) \frac{4 N_{o}}{\sqrt[4]{0.5 \bar{P}_{e, i}}}\left(\frac{10^{\left(\frac{P L_{o}}{10}\right)}}{d_{o}^{n_{i}}}\right) d^{n_{i}} 10^{\left(\frac{S_{i}}{10}\right)}
$$

\section{Set-3}

Using the same methods mentioned in Section 4.1, the upper bound of symbol error rate is 


$$
\bar{P}_{e, i} \leq\left(\frac{\left(\frac{\bar{P}_{R, i}}{R_{i}}\right)}{2 N_{o}}\right)^{-4}
$$

$i \in\{3\}$. The average power at the receiver antenna required for the target SER $\bar{P}_{e, i}$ is

$$
\bar{P}_{R, i}=\frac{2 R_{i} N_{o}}{\sqrt[4]{\bar{P}_{e, i}}}
$$

The energy efficiency of Set-3 is found by applying (29) to $(11)$

$$
E_{i=3}=\frac{P+\vartheta R_{i}}{R_{i}}+(\xi / \eta) \frac{2 N_{o}}{\sqrt[4]{\bar{P}_{e, i}}}\left(\frac{10^{\left(\frac{P L_{o}}{10}\right)}}{d_{o}^{n_{i}}}\right) d^{n_{i}} 10^{\left(\frac{S_{i}}{10}\right)}
$$

\subsection{Energy Efficiency of Optimized Adaptive WBAN System}

As mentioned in detail in [5-7], in the optimized adaptive system, one set of adaptive schemes is employed over a portion of a frame, and possibly changes to different adaptive schemes for the transmission of the remaining part of that frame. During the $\mathrm{q}^{\text {th }}$ frame transmission, where $q=0,1,2, \ldots, Q-1$, and $Q$ is the number of frames, a full body rotation, i.e. $360^{\circ}$, may generate several frame transmissions, depending on the rotation speed. Performance of the adaptive WBAN system in terms of energy efficiency depends on the selected adaptive scheme Set-i on a frame-to-frame basis. Denote the connectivity between a body worn transceiver (or hub) and the external access point $C_{i}^{q}$ at a certain body direction as

$$
C_{i}^{q}=\left\{\begin{array}{lr}
1, & \text { if Set } i \text { is selected on the } \mathrm{q}^{\text {th }} \text { frame } \\
0, & \text { otherwise }
\end{array}\right.
$$

The traffic (or data rate) $R_{i}^{q}$ in the associated link is defined as

$$
R_{i}^{q} \leq B \Gamma_{i}^{q}
$$

where $\Gamma_{i}^{q}$ is the spectral efficiency of Set-i of the $\mathrm{q}^{\text {th }}$ frame, and $B$ is the system bandwidth. Thus, average energy efficiency per frame $\bar{E}_{T}$ of the adaptive WBAN system is calculated as

$$
\bar{E}_{T}=\frac{1}{Q} \sum_{q=0}^{Q-1} \sum_{i}^{3} C_{i}^{q} E_{i}^{q}=\frac{1}{Q} \sum_{q=0}^{Q-1} \sum_{i}^{3} C_{i}^{q} \frac{P_{T o t}\left(\Gamma_{i}^{q}\right)}{B \Gamma_{i}^{q}}
$$

where $E_{i}^{q}$ is the energy efficiency of Set-i of the $\mathrm{q}^{\text {th }}$ frame.
In order to minimize the energy consumption, given the symbol error rate (SER) not exceeding the target SER, the optimized adaptive scheme is to be selected appropriately. This optimization process is equivalent to minimizing the average energy efficiency per frame $\bar{E}_{T}$

$$
\begin{gathered}
\min _{i \in\{1,2,3\}} \bar{E}_{T}=\min _{i \in\{1,2,3\}} \frac{1}{Q} \sum_{\substack{q=0 \\
\text { s.t. }}}^{Q-1} \sum_{i}^{3} C_{i}^{q} \frac{P_{T o t}\left(\Gamma_{i}^{q}\right)}{B \Gamma_{i}^{q}} \\
\sum_{i} C_{i}^{q}=1, \forall i \in\{1,2,3\}, \quad C_{i}^{q} \in[0,1] \\
\Gamma_{i}^{q} \in \Gamma, \text { where } \Gamma=\{1,2,3\} \\
\sum_{q=0}^{Q-1} \sum_{i=1}^{3} \bar{E}_{T} R_{i}^{q} \leq \text { available energy }
\end{gathered}
$$

From (34) it can be seen that $\min _{i \in\{1,2,3\}} \bar{E}_{T}$ can be found by selecting the minimum value of $\sum_{i=1}^{3} C_{i}^{q} \frac{P_{T o t}\left(\Gamma_{i}^{q}\right)}{B \Gamma_{i}^{q}}$ in each frame transmission. In other word, minimizing the average energy efficiency of the proposed adaptive WBAN system is equivalent to selecting an adaptive scheme from three possible schemes which generates minimum energy consumption to be employed in the current frame and each subsequent frame, given the target SER is met. Because there are only three possible adaptive schemes to choose in every frame transmission as given by the constraint in (36), thus the exhaustive search can be utilized without implying a hefty computational load [28]. It is important to note that the total energy consumed during all frames transmission must not exceed the available energy provided by the energy resource (e.g. battery), as given by Eq. (37).

\section{Relation between Energy Efficiency and Spectral Efficiency in Adaptive WBAN System}

Energy efficiency is a critical issue in WBAN design due to limited power resources of WBAN devices or nodes and impracticality to replace batteries frequently, particularly the implant nodes. As a matter of fact, increasing energy efficiency always conflicts with improving spectral efficiency [26], [27]. Therefore, energy efficiency and spectral efficiency cannot be optimized simultaneously [26]. Luckily the proposed adaptive WBAN system has high capacity compared to the capacity requirement of the current and foreseeable future WBAN applications [1], [5]. Therefore, in [6-7], we have optimized the BER performance of the proposed adaptive WBAN system, given the capacity as constraint, without taking the energy consumption issue into consideration. This optimization gave us a favorable BER 
performance compared to the performance of the nonadaptive WBAN system.

In order to understand the behavior of the energy consumption vs spectral efficiency in the proposed adaptive WBAN system, the following sections provide the analysis of this relation.

\subsection{I10 configuration}

The spectral efficiency of the $2 \mathrm{I} 1 \mathrm{O}$ configuration in bps/Hz when the channel coefficients are unknown to the transmitter [27 eq. (4.30)] is

$$
\Gamma_{2 \mathrm{I} 10}=\log _{2}\left(1+\frac{E_{S}}{M_{T} N_{o}}\|\boldsymbol{h}\|_{F}^{2}\right)
$$

where $\boldsymbol{h}$ is the channel vector, and $\|\cdot\|_{F}$ is the Frobenius operation. $\|\boldsymbol{h}\|_{F}^{2}$ is assumed to be equal 1. From the link budget where $G=1 / P L$ (i.e. $G(d B)=-P L(d B)$ ), following Eq. (9) and using the relation in (7) and (10), this equation is held

$$
\Gamma_{2 \mathrm{I} 10}=\log _{2}\left(1+\frac{G(\eta / \xi) P_{P A}}{M_{T} B N_{o}}\right)
$$

Denote $\Gamma_{2 \mathrm{I} 10}=\Gamma_{i}$, then $P_{P A}=\frac{M_{T} B N_{o}}{G}(\xi / \eta)\left(2^{\Gamma_{i}}-1\right)$. Recall that the energy efficiency is defined by ratio between total energy consumption and data rate as in Eq. (5). Thus, the energy efficiency as a function of the spectral efficiency of the adaptive WBAN system in the $2 \mathrm{I} 1 \mathrm{O}$ configuration can be found by applying $P_{P A}$ of (39) to (5) and (8)

$$
E_{i, 2 \mathrm{I} 1 \mathrm{O}}\left(\Gamma_{i}\right)=\frac{P+\vartheta B \Gamma_{i}}{B \Gamma_{i}}+\frac{M_{T} N_{o}}{G}(\xi / \eta) \frac{\left(2^{\Gamma_{i}}-1\right)}{\Gamma_{i}}
$$

\subsection{0 configuration}

The maximum spectral efficiency of orthogonal $2 \mathrm{I} 2 \mathrm{O}$ channels in $\mathrm{bps} / \mathrm{Hz}$ when the channel coefficients are unknown to the transmitter [27, eq. (4.33)] is

$$
\Gamma_{2 \mathrm{I} 2 \mathrm{O}}=M \log _{2}\left(1+\frac{E_{s}}{N_{o}}\right)
$$

where $M=M_{T}=M_{R}$. From the link budget, the spectral efficiency of $2 \mathrm{I} 2 \mathrm{O}$ is

$$
\Gamma_{2 \mathrm{I} 2 \mathrm{O}}=M \log _{2}\left(1+\frac{G(\eta / \xi) P_{P A}}{B N_{o}}\right)
$$

Denote $\Gamma_{2 \mathrm{I} 2 \mathrm{O}}=\Gamma_{i}$, thus $P_{P A}=\frac{B N_{O}}{G}(\xi / \eta)\left(2^{\Gamma_{i} / M}-1\right)$. As a result, by applying the similar way as in Section 5.1, the energy efficiency as a function of spectral efficiency of the adaptive WBAN system in the $2 \mathrm{I} 2 \mathrm{O}$ configuration can be found by applying $P_{P A}$ of (42) to (5) and (8)

$$
E_{i, 2 \mathrm{I} 20}\left(\Gamma_{i}\right)=\frac{P+\vartheta B \Gamma_{i}}{B \Gamma_{i}}+\frac{N_{o}}{G}(\xi / \eta) \frac{\left(2^{\frac{\Gamma_{i}}{M}}-1\right)}{\Gamma_{i}}
$$

\section{Numerical Results}

In this section, a numerical evaluation is provided to assess how well the proposed adaptive WBAN system performs in terms of energy efficiency. As stated before, the energy efficiency is measured by the average energy consumption per bit on a frame-to-frame basis, either for transmission energy only or the total energy consumption of the system. This energy consumption per bit is frame independent, i.e. not depending on the frame size (c.f. Section 4.3). Accordingly, higher energy consumption per bit implies a less energy-efficient system.

The system uses a MB-OFDM UWB standard with 528 $\mathrm{MHz}$ bandwidth [4]. The spectral efficiency of Set-1, Set2 , and Set-3 are $3 \mathrm{bps} / \mathrm{Hz}, 2 \mathrm{bps} / \mathrm{Hz}$, and $1 \mathrm{bps} / \mathrm{Hz}$, respectively [5-7]. The maximum distance between a hub placed on the torso and a WAP is $5 \mathrm{~m}$. With this distance, the maximum transmission delay is around $16 \mathrm{~ns}$, which is negligible. The movement of body directions changes the hub's transceiver direction towards the WAP, resulting in different fading channels. It is assumed that a perfect channel state estimation is available at the receiver, and channel coefficients are assumed to be constant during transmission of each STFC block, but random between consecutive STFC blocks [27], [29]. The path loss model and parameters are based on the IEEE's UWB WBAN CM3 and CM4 models, i.e. log-normal shadowing [22]. The other parameters are listed in Table 2 and quoted from the approaches found in [3], [8] and [9] for the sake of illustration and performance comparison between the adaptive WBAN and non-adaptive one.

Table 2. Numerical Properties

\begin{tabular}{ll}
\hline \hline Parameters & Value \\
\hline$\vartheta$ constant & $10^{-11}$ \\
Rate-independent power & $80 \mathrm{~mW}$ \\
PAPR & 1 \\
Drain efficiency of PA & 0.35 \\
Target SER & $10^{-3}$ \\
\hline \hline
\end{tabular}

It is assumed that the $2 \mathrm{I} 2 \mathrm{O}$ transceiver has double the circuitry of the $2 \mathrm{I} 1 \mathrm{O}$ one. As a consequence, given the transceiver has the same structure, the circuitry power consumption in $2 \mathrm{I} 2 \mathrm{O}$ also doubles compared to that in 2I1O. As mentioned earlier, the rate-dependent power is modelled to increase linearly with respect to the rate, i.e. $\mathbf{P}\left(\mathbf{R}_{\mathbf{i}}\right) \sim \boldsymbol{\vartheta} \mathbf{R}_{\mathbf{i}}$. Note that the rate is equal to the spectral 
efficiency of the adaptive WBAN system multiplied by the bandwidth. This rate-dependent and the rateindependent power make the circuitry power consumption increase linearly as the spectral efficiency increases.

The transmission power of each adaptive scheme used in the adaptive WBAN system is evaluated. Powers measured in terms of transmission energy per bit at the power amplifier (PA) of each adaptive scheme as a function of the distance in $2 \mathrm{I} 1 \mathrm{O}$ and $2 \mathrm{I} 2 \mathrm{O}$ are depicted in Figure 3 and Figure 4, respectively (refer to the second part of Eqs. (20), (23), (27), and (30)). The dash-dot magenta line is the power emitted by PA in Set-1 mode, while the dash blue line and the solid red line are for Set-2 mode and Set-3 mode, respectively.

It can be seen that in both MIMO configurations the power consumption of PA increases substantially with the distance. However, the lower the rate, (Set-3 has the lowest rate), the less the PA power consumption. Up to the distance of $1 \mathrm{~m}$, the power consumed in PA is insignificant, then it starts to increase quickly afterward. Clearly, the impact of spectral efficiency on the PA power consumption is very substantial, particularly in the larger distance. For example, at the distance of $4 \mathrm{~m}$, the difference between the power consumption of PA in the Set-1 is $133 \%$ larger than in Set-3 in the $2 \mathrm{I} 1 \mathrm{O}$ configuration, and $47 \%$ larger in the $2 \mathrm{I} 2 \mathrm{O}$ configuration as shown in Table 3.

By comparing Figure 3 and Figure 4, the PA power consumed in the $2 \mathrm{I} 1 \mathrm{O}$ adaptive WBAN is considerably higher compared to that in 2I2O. For instance, at the distance of $4 \mathrm{~m}$, the PA power consumption in $2 \mathrm{I} 1 \mathrm{O}$ is up to $382 \%(5.8 \mathrm{~dB})$ higher than in $2 \mathrm{I} 2 \mathrm{O}$ as can be seen in Table 3. That means the adaptive WBAN system with a $2 \mathrm{I} 2 \mathrm{O}$ configuration is more energy-efficient in terms of transmission power required to achieve the same target SER compared to the $2 \mathrm{I} 1 \mathrm{O}$ one. This is because in the orthogonal MIMO system, the diversity order is equal to $M_{T} \times M_{R}$, and the array gain is equivalent to $10 \log _{10} M_{R}$ (dB) [23], [27]. Thus, the 2I2O has a diversity order of 4 and array gain of 2, while the $2 \mathrm{I} 1 \mathrm{O}$ has a diversity order of 2 and no array gain. A higher diversity order means a better capability to mitigate the fading and improve the link reliability, while having an array gain result in a smaller SNR required to obtain the desired SER. The combined impact of these higher diversity order and array gain result in considerably less energy to transmit in the 2I2O adaptive WBAN system to achieve the same target SER as in $2 \mathrm{I} 1 \mathrm{O}$.

Table 3. Transmission Energy per Bit Comparison between 2110 and 2I2O at Distance $4 \mathrm{~m}$.

\begin{tabular}{|c|c|c|c|}
\cline { 2 - 4 } \multicolumn{1}{c|}{} & $\mathbf{2 l 1 0}$ & $\mathbf{2 l 2 O}$ & $\mathbf{2 l 2 0}$ vs 2I10 \\
\hline Set-i & $\begin{array}{l}\text { Transmission } \\
\text { energy (J/bit) }\end{array}$ & $\begin{array}{l}\text { Transmission } \\
\text { energy (J/bit) }\end{array}$ & Improvement \\
\hline Set-1 & $8.19 \mathrm{e}-10$ & $2.14 \mathrm{e}-10$ & $382 \%(5.8 \mathrm{~dB})$ \\
\hline Set-2 & $5.27 \mathrm{e}-10$ & $1.78 \mathrm{e}-10$ & $296 \%(4.7 \mathrm{~dB})$ \\
\hline Set-3 & $3.51 \mathrm{e}-10$ & $1.45 \mathrm{e}-10$ & $242 \%(3.8 \mathrm{~dB})$ \\
\hline
\end{tabular}

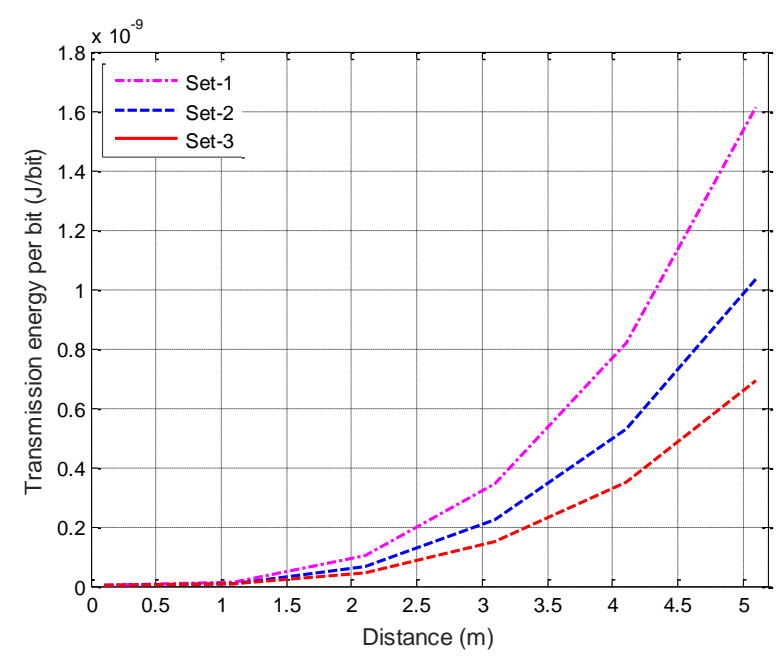

Figure 3. Transmission energy of each adaptive scheme as a function of distance between the hub and the WAP in 2110 configuration.

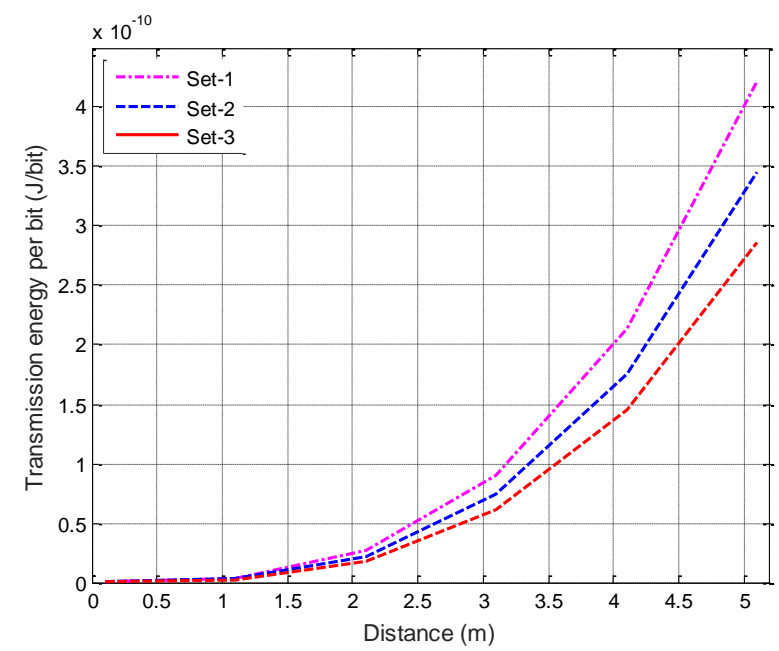

Figure 4. Transmission energy of each adaptive scheme as a function of distance between the hub and the WAP in $212 \mathrm{O}$ configuration.

Figure 3 and Figure 4 just compare the transmission power consumption between three adaptive schemes used in the adaptive WBAN systems. Due to a small distance between the hub and the WAP in typical WBAN implementations, beside the transmission power, the circuitry power consumption also plays an important role in the total power consumption. Thus, it would be more comprehensive to compare the energy efficiency of those systems by taking the total energy consumption into consideration.

Figure 5 and Figure 6 present the total energy consumption per bit, which include both transmission and circuitry power consumptions, as a function of distance of the adaptive WBAN compared to the non-adaptive one in the $2 \mathrm{I} 1 \mathrm{O}$ and $2 \mathrm{I} 2 \mathrm{O}$ configurations, respectively. It can be seen that the adaptive WBAN system outperforms the 


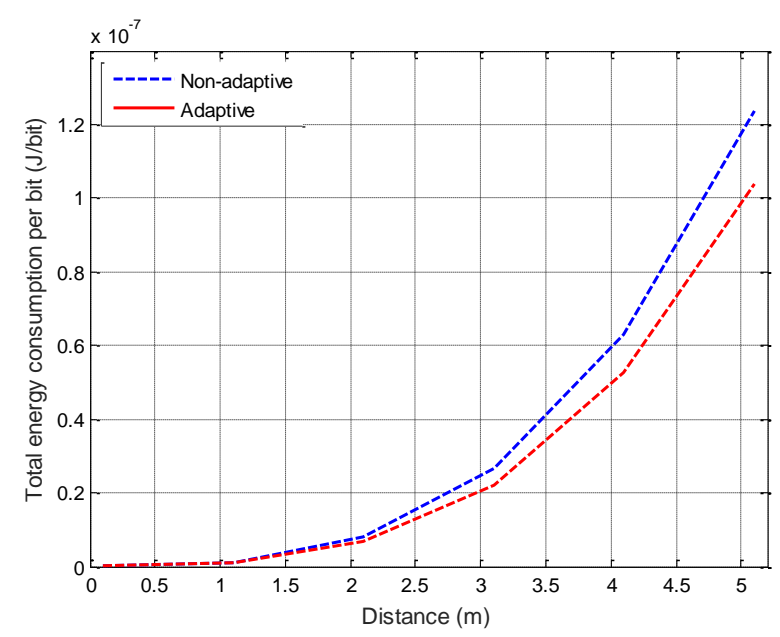

Figure 5. Comparison of energy efficiency as a function of distance between the non-adaptive and the adaptive WBAN systems in 2110.

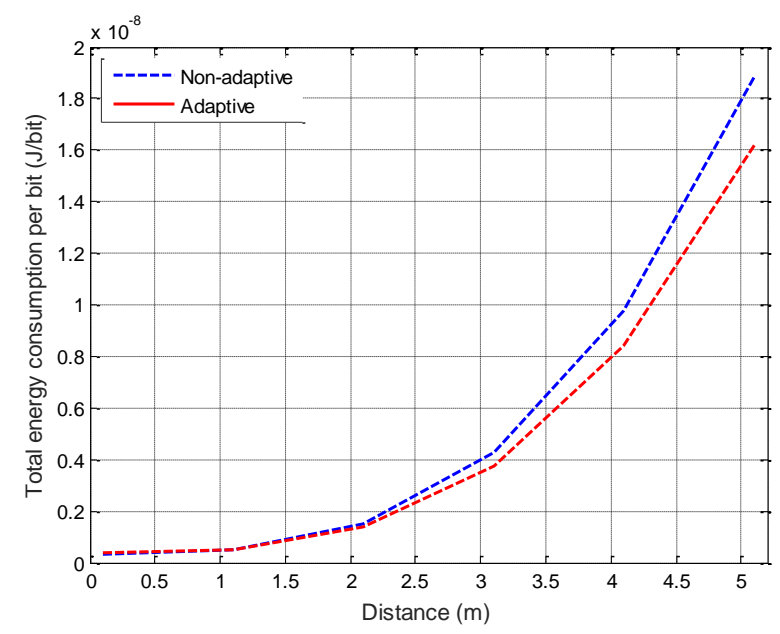

Figure 6. Comparison of energy efficiency as a function of distance between the non-adaptive and the adaptive WBAN systems in $2 \mathrm{I} 2 \mathrm{O}$.

non-adaptive one in terms of energy efficiency in both MIMO configurations for the distance between hub and WAP greater than $1 \mathrm{~m}$. The larger the propagation distance, the larger the difference between energy consumption per bit in the two configurations.

Table 4 shows the total energy saving by employing adaptive scheme as compared to non-adaptive one, and improvement by utilizing $2 \mathrm{I} 2 \mathrm{O}$ compared to $2 \mathrm{I} 1 \mathrm{O}$ at distance $4 \mathrm{~m}$. For example, to obtain the same target SER at the distance of $4 \mathrm{~m}$, the $2 \mathrm{I} 1 \mathrm{O}$ adaptive WBAN system, consumes $19.1 \%$ less energy per bit compared to the nonadaptive WBAN system. Meanwhile, the $2 \mathrm{I} 2 \mathrm{O}$ adaptive WBAN system consumes $15.5 \%$ less energy per bit compared to the non-adaptive WBAN.

From Figure 5 and Figure 6 (and Table 4), it can also be observed that the $2 \mathrm{I} 2 \mathrm{O}$ adaptive WBAN system at a distance of $4 \mathrm{~m}$ is $7.9 \mathrm{~dB}$ superior compared to the $2 \mathrm{I} 1 \mathrm{O}$ one in terms of energy efficiency, despite of the fact that the $2 \mathrm{I} 2 \mathrm{O}$ consumes twice as much power in its circuitry compared to the 2I1O. When energy consumption in both circuitry and PA are considered, the 2I2O adaptive WBAN system (c.f. Figure 6) consume around $626 \%$ or $7.9 \mathrm{~dB}$ less energy per bit compared to the $2 \mathrm{I} 1 \mathrm{O}$ one (c.f. Figure 5). The same is true for the non-adaptive one. It is a huge energy saving, given the fact that the system employs a very high data rate. This fact illustrates clearly the advantages of having a 2I2O MIMO employed in the adaptive WBAN system as elaborated earlier.

Table 4. Comparison between Energy Efficiency of Adaptive and Non-adaptive WBAN System in 2110 and $2 \mathrm{I} 2 \mathrm{O}$ at Distance $4 \mathrm{~m}$.

\begin{tabular}{|c|c|c|c|}
\hline & 2110 & 2120 & 2120 vs 2110 \\
\hline Scheme & $\begin{array}{c}\text { Total energy } \\
\text { (J/bit) }\end{array}$ & $\begin{array}{c}\text { Total energy } \\
\text { (J/bit) }\end{array}$ & Improvement \\
\hline Adaptive & $5.28 e-8$ & $8.43 e-9$ & $\begin{array}{c}626 \% \\
(7.9 \mathrm{~dB})\end{array}$ \\
\hline $\begin{array}{l}\begin{array}{l}\text { Non- } \\
\text { adaptive }\end{array} \\
\end{array}$ & $6.29 e-8$ & $9.74 e-9$ & $\begin{array}{c}645 \% \\
(8.1 \mathrm{~dB}) \\
\end{array}$ \\
\hline $\begin{array}{l}\text { Adaptive vs } \\
\text { Non-adaptive } \\
\text { energy saving }\end{array}$ & $19.1 \%$ & $15.5 \%$ & \\
\hline
\end{tabular}

Figure 7 describes the relation between energy consumption and spectral efficiency of the adaptive WBAN system with $2 \mathrm{I} 1 \mathrm{O}$ and $2 \mathrm{I} 2 \mathrm{O}$ configurations at a fixed path loss (i.e. at distance $d=4 \mathrm{~m}$ ). It is important to note that the spectral efficiency range, for both MIMO configurations shown in that Figure, are within Shannon's capacity limit, as calculated by Eq. (38) and Eq. (41).

At very low spectral efficiency (i.e. close to zero), the energy consumption is roughly equal to the total energy dissipated in the circuitry, because the transmission power is very low at a very low bit rate. Therefore, the energy consumption per bit is initially high (since the bit rate is very low or close to zero, refer to Eq. (5)). As the spectral efficiency increases up to $2 \mathrm{bps} / \mathrm{Hz}$, the energy consumption per bit of both MIMO configurations decreases substantially as the bit rate goes up. In fact, the transmission energy starts to pick up at this spectral efficiency range. But, the energy consumed in the circuitry still dominates the transmission energy due to the small distance between the hub and the external WAP. As a result of the double circuitry of the $2 \mathrm{I} 2 \mathrm{O}$ configuration compared to the $2 \mathrm{I} 1 \mathrm{O}$ one, it is obvious that the $2 \mathrm{I} 2 \mathrm{O}$ configuration consumes more energy per bit in this low spectral efficiency region, hence possessing lower energy efficiency compared to the $2 \mathrm{I} 1 \mathrm{O}$ one.

From the spectral efficiency of $2 \mathrm{bps} / \mathrm{Hz}$ onward, the energy consumption per bit in the $2 \mathrm{I} 1 \mathrm{O}$ configuration increases much more rapidly than $2 \mathrm{I} 2 \mathrm{O}$. In other words, the energy efficiency of the former decreases faster than that in the latter. At least three factors contribute to this phenomenon, namely the increase of the transmission energy consumption, the diversity order, and the array gain. First, the dominance of energy consumption in circuitry diminishes and the transmission energy 


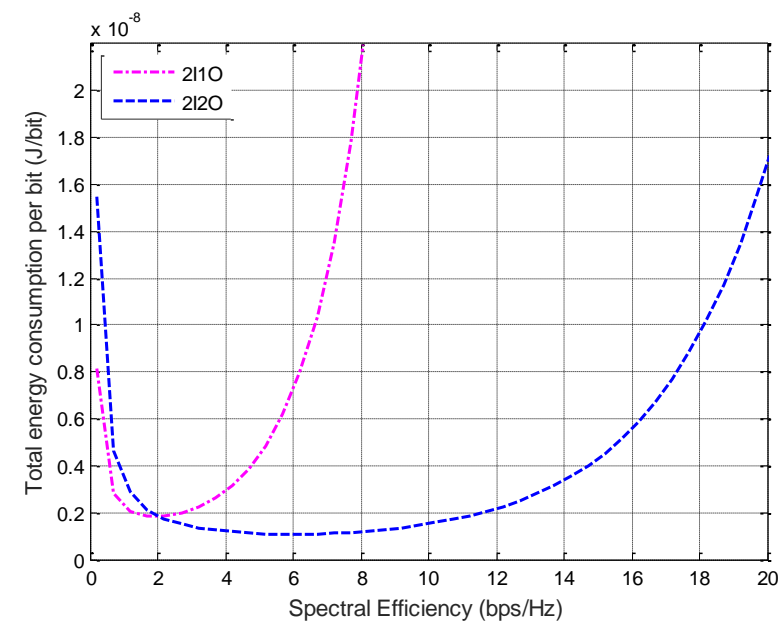

Figure 7. Energy efficiency as a function of spectral efficiency in the adaptive WBAN system for two different MIMO configurations, $d=4 \mathrm{~m}$.

consumption rises. Increasing spectral efficiency means more transmission power is required. The higher the spectral efficiency is, the higher the energy consumption per bit is (therefore lower energy efficiency). Further, the diversity order and array gain have significant impacts on the system. As mentioned previously, the $2 \mathrm{I} 2 \mathrm{O}$ possesses a higher diversity order than the $2 \mathrm{I} 1 \mathrm{O}$, which means it has a better capability to mitigate the fading and improve the link reliability. In addition, the array gain of the $2 \mathrm{I} 2 \mathrm{O}$ results in a smaller required SNR to obtain a desired SER. The combined impact of these two factors results in a considerably less energy needed to transmit the signals. This phenomenon can be seen in Figure 7, that because of having no array gain and a lower diversity order, the energy consumption per bit in $2 \mathrm{I} 1 \mathrm{O}$ increases in much faster pace compared to $2 \mathrm{I} 2 \mathrm{O}$ when the spectral efficiency increases. Meanwhile, spectral efficiency only slowly increases the energy consumption per bit in 2I2O. In fact, the energy consumption per bit in this case relatively flats from spectral efficiency of $2 \mathrm{bps} / \mathrm{Hz}$ to $12 \mathrm{bps} / \mathrm{Hz}$. Thus, the $2 \mathrm{I} 2 \mathrm{O}$ adaptive WBAN system delivers a much better energy efficiency compared to the $2 \mathrm{I} 1 \mathrm{O}$ one. These facts also suggest that employing higher rate STFC code to obtain a higher spectral efficiency or capacity in the $2 \mathrm{I} 2 \mathrm{O}$ adaptive WBAN system is possible without suffering from very high energy consumption.

\section{Conclusion}

In this paper, we investigate the energy efficiency of the proposed adaptive WBAN system, in comparison with the non-adaptive one working at the same WBAN environment and scenario. Due to a very small distance between the hub and the WAP in typical WBAN implementations, the circuitry power consumption plays a significant role in the total power consumption, apart from the transmission power. It is found that the adaptive
WBAN system outperforms the non-adaptive one in terms of energy efficiency in both $2 \mathrm{I} 1 \mathrm{O}$ and $2 \mathrm{I} 2 \mathrm{O}$ configurations. Furthermore, as investigated in [6] and [7], the adaptive WBAN system is also superior to the non-adaptive one in terms of bit error rate performance. This better energy efficiency coupled with the improved BER performance indicates that the proposed adaptive WBAN scheme is an attractive physical layer of WBAN system for mHealth services. Thus, this scheme offers not only better performance, but also provides energy saving to crucially prolong battery life of a WBAN system.

\section{Acknowledgment}

The first author acknowledges and is grateful to the financial support from AusAID through the Australian Development Scholarship (ADS) scheme. The authors appreciate all feedbacks from anonymous reviewers.

\section{References}

[1] Cavallari, R., et. al. (2014) A survey on wireless body area networks: Technologies and design challenges. IEEE Communication Survey and Tutorial, vol. 99 (3):1635-1657.

[2] IEEE Computer Society (2012) IEEE standard for local and metropolitan area networks-Part 15.6: Wireless Body Area Networks, (New Jersey:IEEE).

[3] Yang, W.B. and Sayrafian-Pour, K. (2012) Interference mitigation using adaptive scheme in body area networks. Int J. Wireless Inf. Networks, vol. 19: 193-200.

[4] Final Deliverable 1.5 (2009), Multiband OFDM physical layer specification, (WiMedia Alliance).

[5] Sudjai, M. and Tran, L.C. (2014) A BER based adaptive STFC MB-OFDM UWB system for WBAN applications. In Proc. IEEE International Conference on Communication (ICC), Sydney, Jun. 2014, pp. 5681-5686,

[6] Sudjai, M., Tran, L.C., Safaei, F. and Phung, S.L. (2014) Optimal adaptive wireless body area networks for high speed mHealth services. W. de Gruyter Journal on Biomedizinische Technik, vol. 59 (s1): 750-753.

[7] Sudjai, M., Tran, L.C., Safaei, F., Wysocki, T. and Phung, S.L. (2016) High Speed Adaptive Wireless Body Area Networks. Under review in EURASIP Journal on Wireless Communications and Networking.

[8] Cui, S., Goldsmith, A. and Bahai, A. (2005) Energy-constrained modulation optimization. IEEE Trans. on Wireless Commun., vol.4 (5): 2349-2360.

[9] Wang, T. and Vandendorpe, L. (2013) On the Optimum Energy Efficiency for Flat-fading Channels with Rate-dependent Circuit Power. IEEE Trans. on Commun., vol. 61 (12): 4910-4921.

[10] Amin, O., et.al. (2014) Energy efficiency and spectral efficiency trade-off for OFDM systems with imperfect channel estimation. In Proc. IEEE Intern. Conference on Commun., Sydney, Jun. 2014, pp. 3559-3564,

[11] Le, N.P., Tran, L.C. and Safaei, F. (2014) Energy-efficiency analysis of per-subcarrier antenna selection with peak-power reduction in MIMO-OFDM wireless systems. In Intern. Journal of Antennas and Propagation, vol. 2014: 1-13.

[12] Cui, S., Goldsmith, A. and Bahai, A.(2004) Energy-Efficiency of MIMO and Cooperative MIMO Techniques in Sensor Networks. IEEE Journal on Select. Areas in Commun., vol. 22 (6):1089-1098.

[13] Garzas, J., Calzon, C.B. and Armada, A.G. (2007) An energyefficient adaptive modulation suitable for wireless sensor networks with SER and throughput constraints. EURASIP Journal on Wireless Commun. and Networking, vol. 2007: 1-7. 
[14] Abouei, J., et.al., (2011) Energy efficiency and reliability in wireless biomedical implant systems. IEEE Trans. on Inform. Tech. in Biomedicine, vol. 15(3): 456-466.

[15] Ding, J., et.al., (2013) Energy-efficient cooperative relay selection for UWB based body area networks. In Proc. IEEE Intern. Conference on UWB (ICUWB), Sydney, Sep. 2013, pp. 97-102.

[16] Huang, X., Shan, H. and Shen, X. (2011) On energy efficiency of cooperative communications in wireless body area networks. In Proc. IEEE WCNC, Cancun, Mar. 2011, pp. 1097-1101.

[17] Wang, H. et.al., (2011) Information-based energy efficient sensor selection in wireless body area networks. In Proc. IEEE Intern. Conference on Commun., Kyoto, Jun. 2011, pp. 1-6,

[18] Fang, G. and Dutkiewicz, E. (2009) BodyMAC: Energy Efficient TDMA-based MAC Protocol for Wireless Body Area Networks. In Proc. ISCIT, Incheon, Sep. 2009, pp. 1455-1459.

[19] Marinkovic, S.J. et.al. (2009) Energy-efficiency low duty cycle MAC protocol for wireless body area networks. IEEE Trans. on Inform. Tech. in Biomedicine, vol. 13 (6): 915-925.

[20] Argyriou, A., Breva, A.C. and Aoun, M.(2014) Optimizing data forwarding from body area networks in the presence of body shadowing with dual wireless technology nodes. IEEE Trans. on Mobile Computing, vol.14(3): 632-645.

[21] Elias, J. (2014) Optimal design of energy-efficient and costeffective wireless body area networks. Elsevier J. Ad Hoc Netw., vol. 13 (Part B): 560-574.

[22] Yazdandoost, K.Y. and Sayrafian-Pour, K. (2010) Channel model for Body area network (BAN). IEEE P802.15-08-0780-12-0006.

[23] Alamouti, S.M. (1998) A simple transmit diversity technique for wireless communications. IEEE Journal on Selected Areas in Commun., vol. 16(8): 1451-1458.

[24] Sezginer, S. and Sari, H. (2007) A High-Rate Full-Diversity 2x2 Space-Time Code with simple Maximum Likelihood decoding. In Proc. ISSPIT, Cairo, Dec. 2007, pp. 1132-1136.

[25] Rappaport, T.S. (1996) Wireless Communication: Principles and Practice. (New York: IEEE Press).

[26] Proakis, J.G. (2001) Digital Communications, 4th ed. (NewYork: McGraw-Hill).

[27] Paulraj, A., Nabar, R. and Gore, D. (2008) Introduction to SpaceTime Wireless Communication. (Cambridge: Cambridge University Press).

[28] Bertsimas, D. and Weismantel, R. (2005) Optimization Over Integers. (Belmont, MA: Dynamic Ideas).

[29] Tran, L.C. and Mertins, A. (2009) Space-Time-Frequency Code implementa-tion in MB-OFDM UWB communications: design criteria and performance. IEEE Trans. Wireless Commun., vol. 8 (2): 701-713. 